\title{
A Study on Sound Characteristics of Home appliances Noise using Psychoacoustics Parameters
}

\author{
Seong-Geon Bae ${ }^{\# 1}$, Won-Hee Lee ${ }^{* 2}$ and Myung-Jin Bae ${ }^{* * 3}$ \\ \#1 Division of Computer Media Information Engineering, Kangnam University, \\ Gugal-dong, Giheung-gu, Yongin-si, Gyeonggi-do, Korea \\ sgbae@kangnam.ac.kr \\ ${ }^{* 2-3}$ Department of Information and Telecommunication Engineering, Soongsil University, \\ 369 Sangdo-Ro, Dongjak-Gu, Seoul, Korea \\ vbluelovev@ssu.ac.kr, ${ }^{3}$ mjbae@ssu.ac.kr
}

Abstract-This research has analyzed characteristics of sound made from different electronic devices. For more objective data analysis psychoacoustics parameters are used. As source of noise coming from different electronic devices may have a direct influence on people at home, the purpose of this research is to what are its main effects and how they appear. Study on psychoacoustics parameters was done through defining noise made from different products.

Keyword - psychoacoustics, Loudness, roughness, sharpness, fluctuation, compressor, electronic devices

\section{INTRODUCTION}

Modern people, who spend most of their time inside tend to live in the artificial noise made from different electronic devices than friendly noise made from the nature. However, we have been ignoring influence of this source of noise on psychology of acoustics due to the convenience. Therefore, it is very important to analyze characteristics of psychoacoustics of each product and the source of nois $\mathrm{e}^{[6][7]}$. This research carried out a thorough investigation on noise from various electronic devices and analyzed the data by using psychoacoustics parameter. Products used in this research are total 5 types, hair dryer, humidifier, vacuum, Kimchi refrigerator, and refrigerator. Measurement and evaluation were done with these 5 products. By identifying influence of each noise on psychoacoustics, this research focuses on analyzing effect of noise on people through various parameters $^{[9]}$.

For this analysis of psychoacoustics parameters of different electronic devices, Chapter 2 studies sources of noise at home and Chapter 3 analyzes and evaluates characteristics of noise through psychoacoustics parameter. Lastly, Chapter 4 makes the final conclusion.

\section{CHARACTERISTICS OF OVERLOADED SIGNAL}

This Chapter introduces about characteristics of electronic devices from acoustic perspective along with the reasons. For hot air coming out from the hair dryer, it is done by making a wind rapidly. Here, noise is made from this strong wind made to cool down inside heater. For vacuum, it sucks in dust or impurities with the strong power created from the motor. At this time, noise is made from the motor used for making a powerful suction. For Kimchi refrigerator, it has compressor like regular refrigerator to maintain a certain temperature. Depending on its capacity, level of noise differs ${ }^{[2][4]}$. However, it has similar level with the one used at home. For refrigerator, frequency of its use is much higher than Kimchi refrigerator and thus, causes most of the noise. The strong fan is used to remove inside air to the outside, causing noise from the rotating power of the $\operatorname{fan}^{[8][10][3][5]}$.

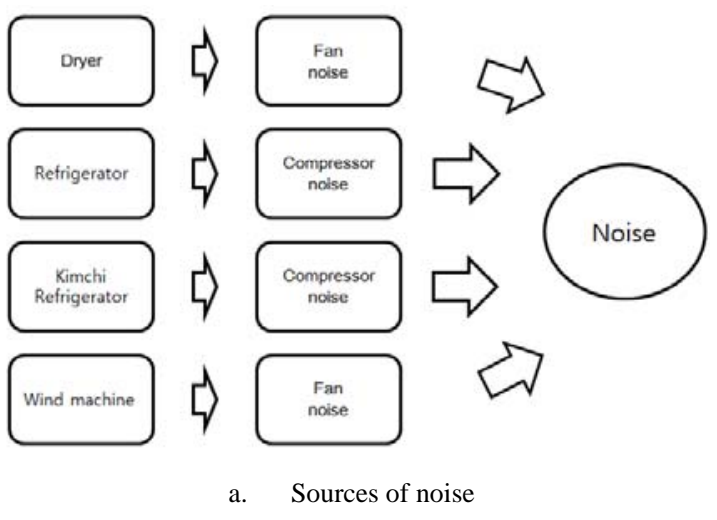




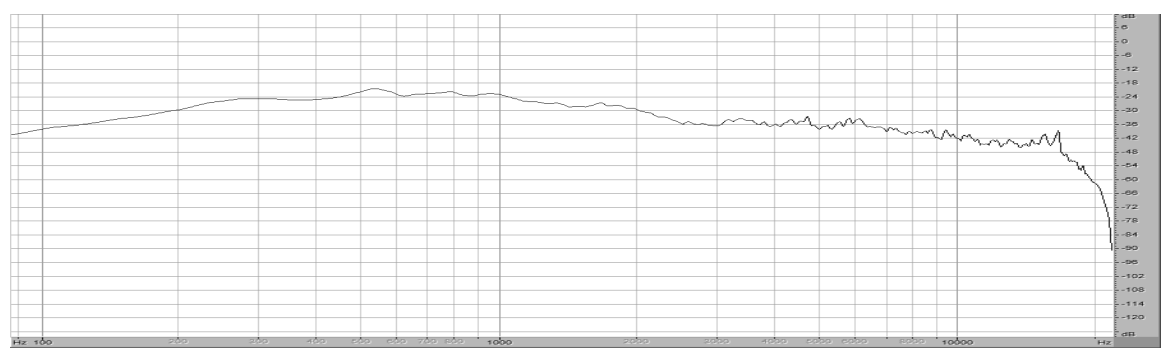

b. General noise frequency of electronic devices

Figure 1. Source of noise

\section{PSYCHOACOUSTICS PARAMETERS}

For parameter, Zwicker parameter was used. Loudness, sharpness, roughness, and fluctuation of each parameter were measured and evaluated. Product noise is measured from its directions ${ }^{[6][8]}$.
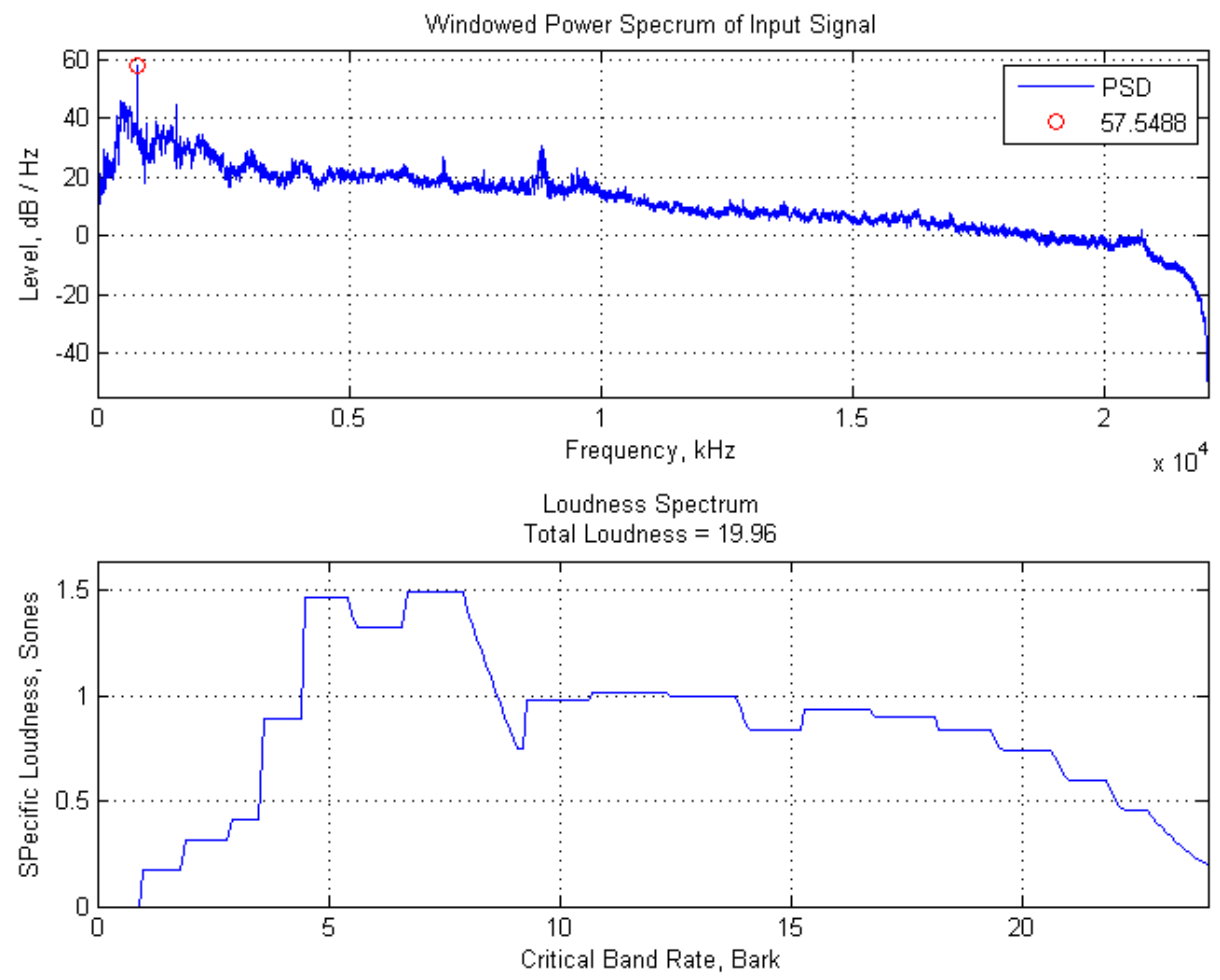

Figure 2. Loudness Parameters of hair dryer

For hair dryer, it had the highest noise at 5 bark and 7 bark. This is because of the noise made from the fan trying to go through a small space. As shown in Figure 2, a smooth line is shown after 7 bark. 

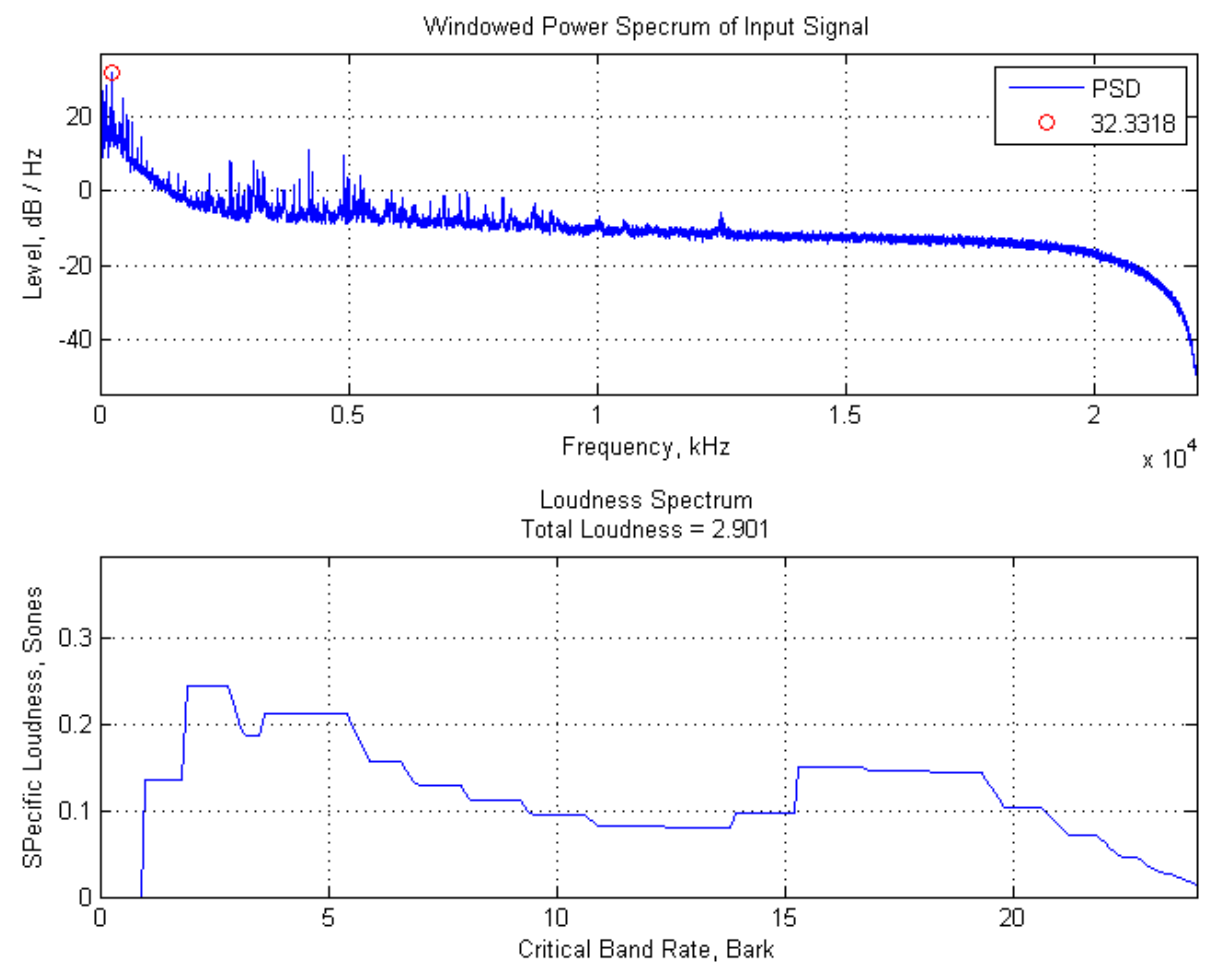

Figure 3. Loudness Parameters of Kimchi refrigerator

Figure 3 is about Kimchi refrigerator, having its highest point at 3 bark. Also, it goes high at 16 bark. Judging from their sizes, it is expected that there are 2 noises, low frequency and middle frequency.
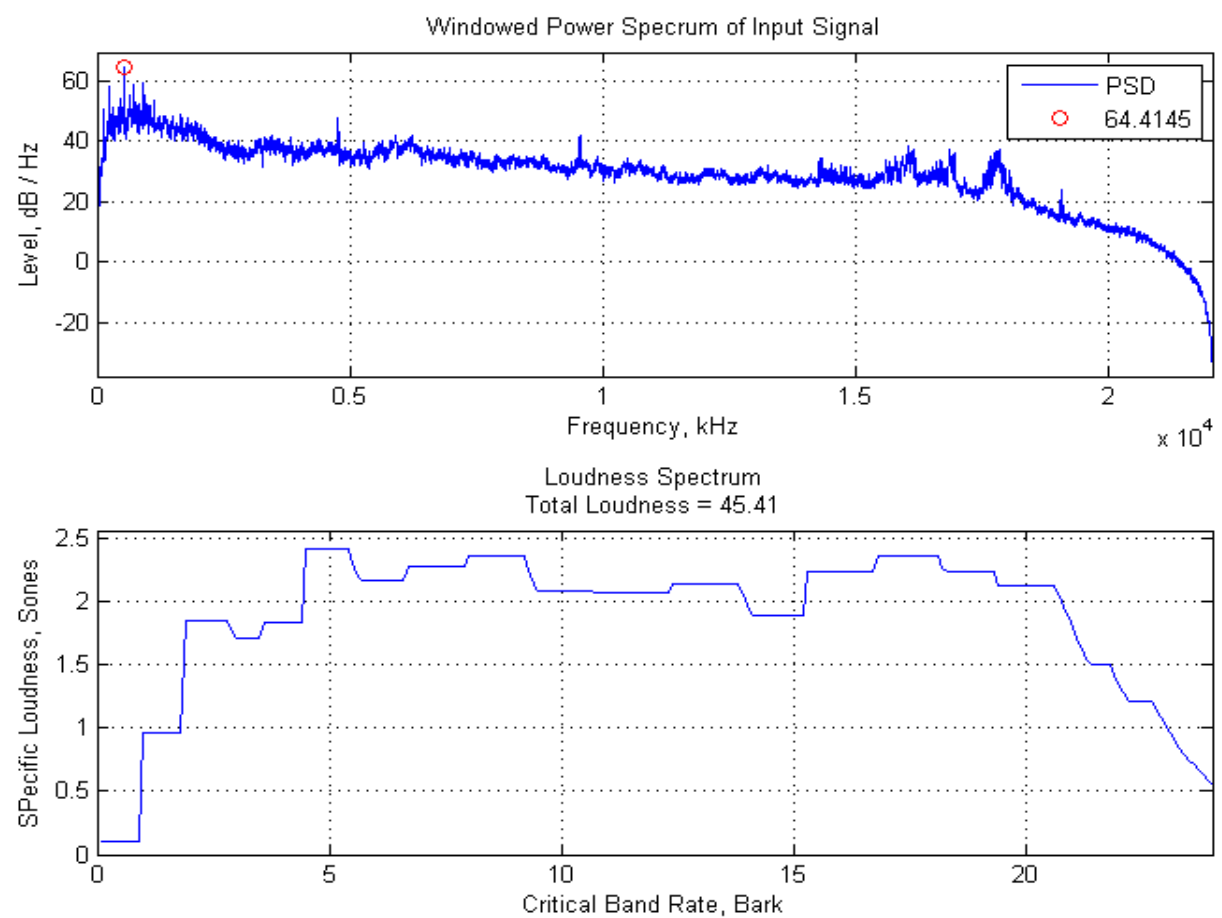

Figure 4. Loudness Parameters of vacuum

Figure 4 shows loudness of vacuum. Loudness tend to be very regular from 5 bark to 17 bark. 

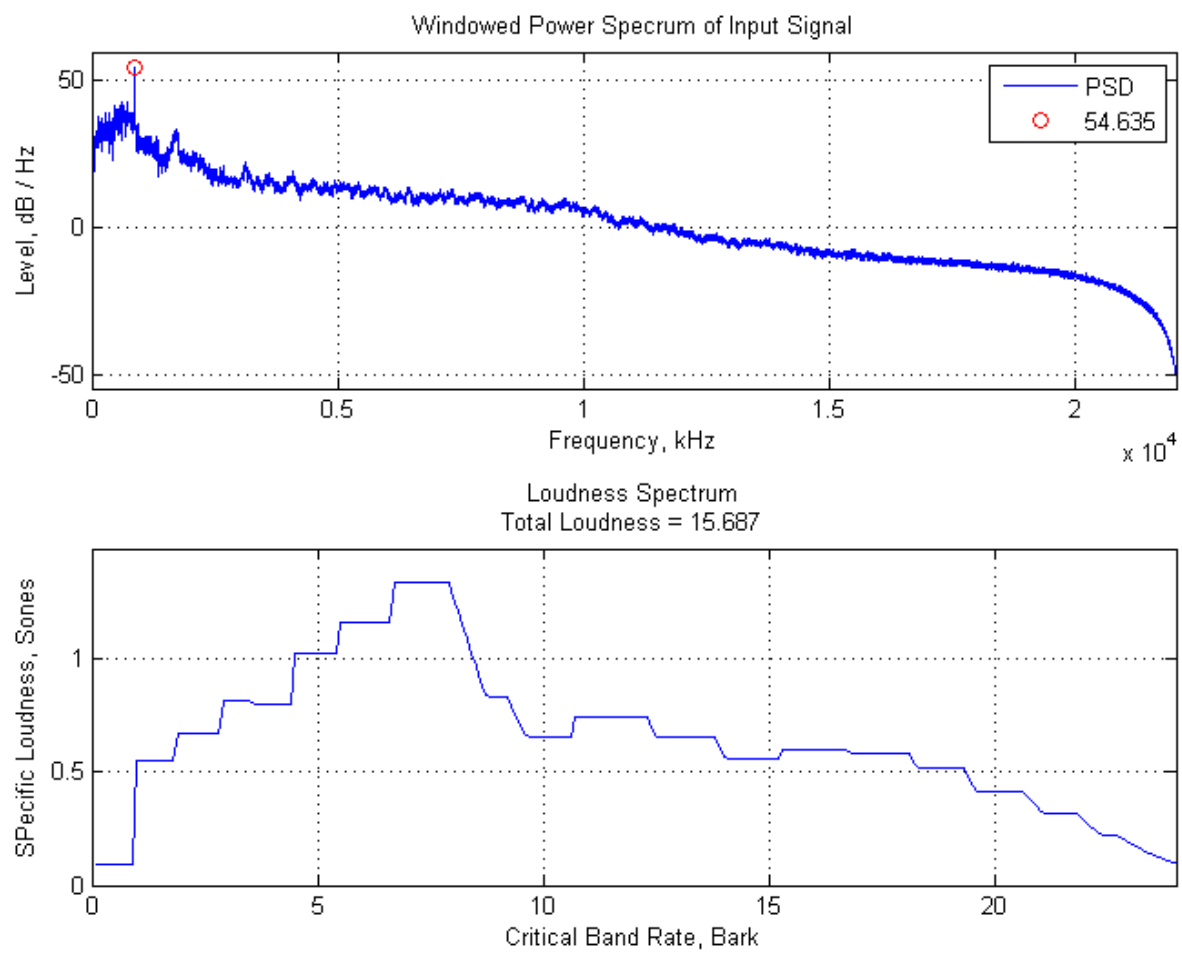

Figure 5. Loudness Parameters of big fan

Figure 5 shows loudness of big fan. It is very concentrated around bark 7. This is because noise is made from the power used to remove wind outside.
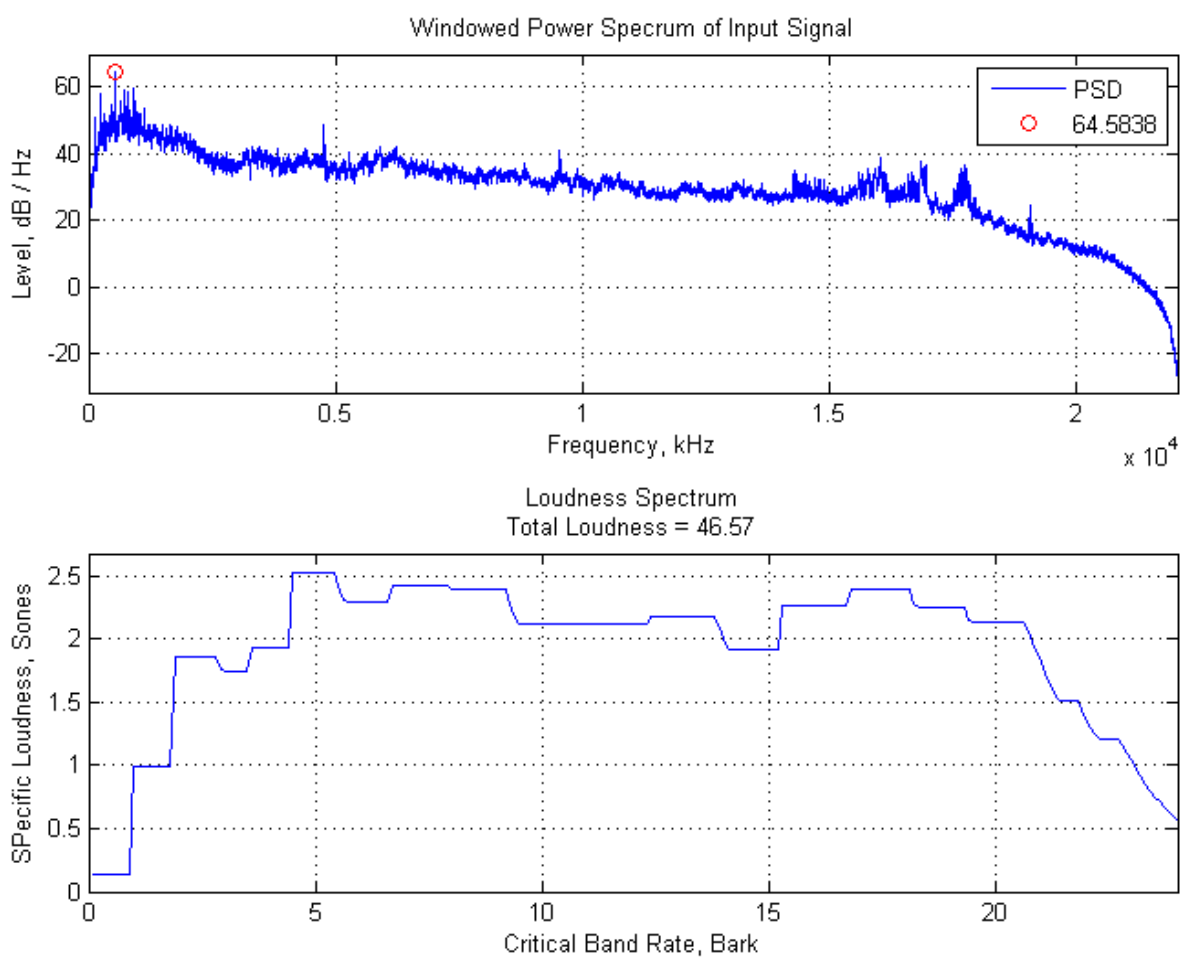

Figure 5. Average Loudness Parameters of electronic devices 
In general, noise in home electronic devices had was high in both 5 bark and 17 bark. However, mostly, they are in a regular shape. This implies that if device is operated at once, noise appears regulars throughout all barks.

Table 1. Psychoacoustics Parameters of electronic devices

\begin{tabular}{llll}
\hline & $\begin{array}{l}\text { Roughness } \\
\text { (asper) }\end{array}$ & $\begin{array}{l}\text { sharpness } \\
\text { (acum) }\end{array}$ & $\begin{array}{l}\text { fluctuation } \\
\text { (vacil) }\end{array}$ \\
Dryer & 0.47 & 2.50 & 0.36 \\
Kimchi Refrigerator & 0.62 & 2.21 & 0.47 \\
Cleaner & 0.39 & 2.67 & 0.29 \\
Wind machine & 0.50 & 2.08 & 0.38 \\
Average & 0.49 & 2.36 & 0.37 \\
\hline
\end{tabular}

According to Table 1, sound changes in $20 \sim 300 \mathrm{~Hz}$ low frequency range, Kimchi refrigerator had the highest roughness and vacuum had the lowest. For sharpness showing sharpness of physical changes, vacuum had the biggest sharpness and big fan had the least. For fluctuation showing regular changes under low frequency of $20 \mathrm{~Hz}$, Kimchi refrigerator had the highest and vacuum had the lowest. This means that vacuum has the minimum changes in its low frequency.

\section{CONCLUSION}

Analysis on acoustic of noise made from different home electronic devices offer an important parameter for finding out influence of noise on people ${ }^{[1][6]}$. Therefore, to have analysis on its characteristics and for more detailed examination, it has studied psychoacoustic parameter. Especially, through analysis of loudness, sharpness, roughness, and fluctuation, various analysis on sound was done ${ }^{[6]}$. For loudness, fan noise was the highest in low frequency and Kimchi refrigerator had the highest roughness. Vacuum had the highest sharpness and Kimchi refrigerator had the highest fluctuation. From this result, we can say that among noise made from different home appliances, compressor of Kimchi refrigerator and fan noise of vacuum give the highest psychological influence on people.

\section{REFERENCES}

[1] Seong-Geon Bae, Won-Hee Lee and Myung-Jin Bae, " A Study on Low Frequency Noise of Dehumidifier using Acoustic Charactristics," IJET Vol.8, No.1, pp.235-237.

[2] Seonggeon Bae, Myungjin Bae, “A New Speech Coding using Harmonics Emphasis Filter,” ISAAC 2013, AACL Vol. 1(2013), pp4344.

[3] Seonggeon Bae, Myungsook Kim, and Myungjin Bae, “On Enhancement Signal Using Non-uniform Sampling in Clipped Signals for LTE Smart Phones”, IEEE ICCE-berlin(2013), pp.125 - 126.

[4] Seonggeon Bae, Hyungwoo Park and Myungjin Bae, "On a New Enhancement of speech Signal using Nonuniform Sampling and Post Filter,”ICHIT 2012, LNCS 7425(2012), pp.723-729, Springer-Verlag.

[5] Geumran Baek, Myungjin Bae, "Study on the Judgment of Intoxication State using Speech Analysis ". Vol.352 CCIS, 2012, pp. $277-$ 282, Springer-Verlag. 2012.

[6] Zwicker, E., Fastl, H. (1990): Psychoacoustics - Facts and Models, Springer - Verlag, Berlin.

[7] Cerrato-Jay G, Collings D, Lowery D. Implementation of sound quality measurements in component rating tests. In: Proceedings of SQS-2002.

[8] Ebbitt G, Davies P. The role of sound quality assessment in noise control. In: Proceedings of NOISE-CON 1998.

[9] Cardozo BL, Veen KG. Estimation of annoyance due to low level sound. Appl Acoust 1979;12(5): 389-96.

[10] ISO 3745. Acoustics - Determination of sound power levels of noise sources using sound pressure - Precision methods for anechoic and hemi-anechoic rooms, 2003.

[11] Tamesue T, Yamaguchi S, Saeki T, Kato Y. A method for prediction of listening score and psychological impression in an actual noise environment. J Sound Vibrat 2005;287:625-36.

\section{AUTHOR PROFILE}

Seong-Geon Bae. He received the Ph.D. degree in Telecommunication \& Information from Soongsil University in 2014. He is currently the Professor of the Division of Computer Media Information at Kangnam University.

Won-Hee Lee. He is currently the under Ph.D. degree in Telecommunication \& Information from Soongsil University.

Myung-Jin Bae. He received the Ph.D. degree in Electronic Engineering from Seoul National University in 1987. He is currently the Professor of the Dept. of Information \& Telecommunication at Soongsil University. 